\title{
Association Between Social Media Use and Cancer Screening Awareness and Behavior for People Without a Cancer Diagnosis: Matched Cohort Study
}

Lei Qin ${ }^{1^{*}}, \mathrm{PhD}$; Xiaomei Zhang ${ }^{1}$, MS; Anlin $\mathrm{Wu}^{2}$, MS; James S Miser ${ }^{3,4,5}$, MD; Yen-Lin Liu ${ }^{4,6,7}, \mathrm{MD}$, PhD; Jason C $\mathrm{Hsu}^{8}, \mathrm{PhD}$; Ben-Chang Shia ${ }^{9,10^{*}}, \mathrm{PhD}$; Linglong $\mathrm{Ye}^{11^{*}}, \mathrm{PhD}$

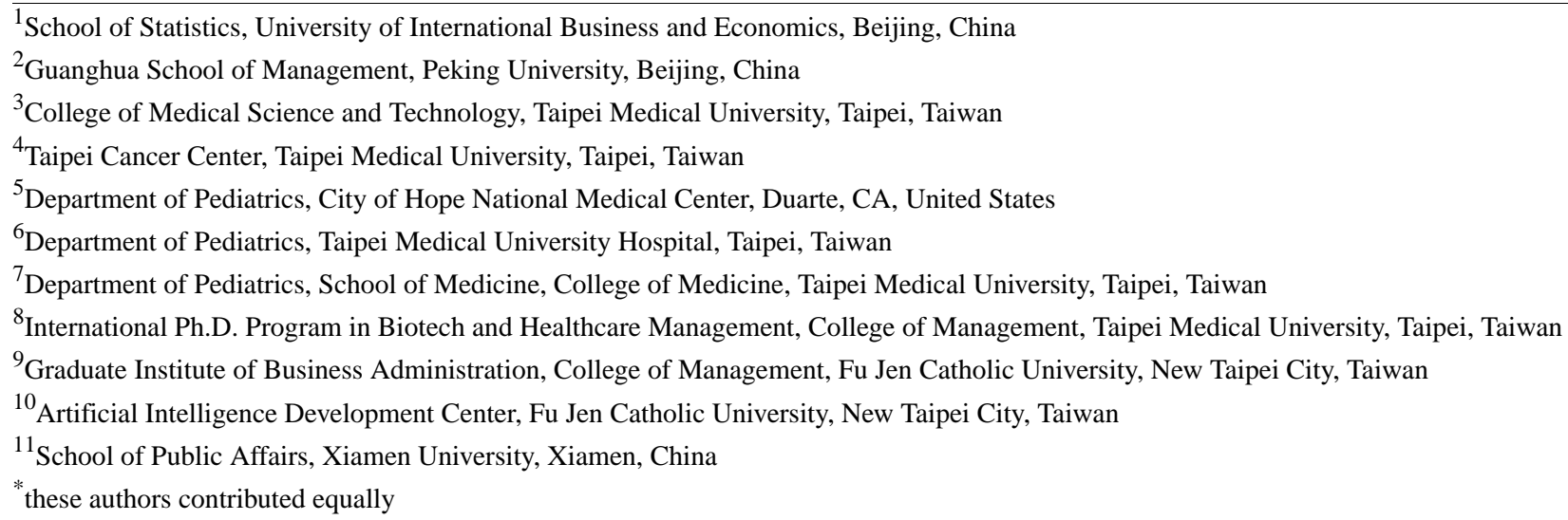

\section{Corresponding Author:}

Linglong Ye, $\mathrm{PhD}$

School of Public Affairs

Xiamen University

No.422, Siming South Road

Xiamen, 361005

China

Phone: 865922182783

Fax: 865922183191

Email: leyloria@gmail.com

\section{Abstract}

Background: The use of social media in communications regarding cancer prevention is rapidly growing. However, less is known about the general population's social media use related to cancer screening awareness and behavior for different cancers.

Objective: We aimed to examine the relationship between social media use and cancer screening awareness and behavior among people without a cancer diagnosis.

Methods: Data were collected from the Health Information National Trends Survey 5 Cycle 1 to 3 in the United States ( $\mathrm{n}=12,227)$. Our study included 10,124 participants without a cancer diagnosis and 3 measures of screening awareness (those who had heard of hepatitis C virus [HCV], human papillomavirus [HPV], and the HPV vaccine) and 4 measures of behavior (those who had prostate-specific antigen tests, Papanicolaou tests for cervical cancer, as well as breast cancer and colon cancer tests). Propensity-score matching was conducted to adjust for the sociodemographic variables between the social media user and nonuser participants. Multivariable logistic regression was used to assess the association of social media use by gender. Jackknife replicate weights were incorporated into the analyses.

Results: Of the 3794 matched participants, 1861 (57.6\% weighted) were male, and the mean age was 55.5 (SD 0.42$)$ years. Compared to social media nonusers, users were more likely to have heard of HCV (adjusted odds ratio [aOR]=2.27, 95\% CI, 1.29-3.98 and $\mathrm{aOR}=2.86,95 \% \mathrm{CI}, 1.51-5.40$, for male and female users, respectively) and $\mathrm{HPV}(\mathrm{aOR}=1.82,95 \% \mathrm{CI}, 1.29-2.58$ and $\mathrm{aOR}=2.35,95 \% \mathrm{CI}, 1.65-3.33$, for male and female users, respectively). In addition, female users were more likely to have heard of the HPV vaccine ( $\mathrm{aOR}=2.06,95 \% \mathrm{CI}, 1.41-3.00)$. No significant associations were found between social media use and 
prostate-specific antigen tests in males, Papanicolaou tests and breast cancer tests in females, or colon cancer tests in both male and female users.

Conclusions: While social media services can potentially promote cancer screening awareness in the general population, but they did not improve screening behavior after adjusting for socioeconomic status. These findings strengthened our understanding of social media use in targeting health communications for different cancers.

(J Med Internet Res 2021;23(8):e26395) doi: 10.2196/26395

\section{KEYWORDS}

social media; cancer screening awareness; cancer screening behavior; gender-specific effects; propensity-score matching; general population

\section{Introduction}

The introduction of screening for early prostate, colorectal, breast, and cervical cancer detection has significantly reduced mortality rates over the past few decades [1]. However, cancer remains the second leading cause of death in the United States [2], and premature cancer deaths resulted in US $\$ 94.4$ billion lost earnings among people aged 16 to 84 years in 2015 [3]. Therefore, there is growing concern regarding the low proportion of cancer screening awareness and behavior in the general population and the relative disparities in cancer screening awareness and behavior due to race and ethnicity and socioeconomic status.

A recent National Health Interview Survey reported that the utilization of recommended cancer screenings is far lower than the Healthy People 2020 targets for the nation [4]. However, cancer screening awareness and behavior disparities exist according to cancer types, race and ethnicity, socioeconomic status, and other health care access factors $[1,4,5]$. For example, from 2005 to 2015, colorectal cancer screenings have increased steadily, but prostate, breast, and cervical cancer screenings have declined [6]. In addition, prostate, cervical, breast, and colon cancer screening rates are lower among Hispanic and Asian groups than non-Hispanic White and Black groups, declining with decreasing education levels [6]. Therefore, new channels for informing target populations are needed to improve the cancer screening awareness and behavior regarding different cancers and reduce the relative disparities.

The use of social media has increased over the past decade and has become a new channel for promoting cancer prevention [7]. Compared to older approaches to cancer interventions with unidirectional and paternalistic outreach barriers, contemporary social media is easy to access, bidirectional, interactive, and patient-driven $[7,8]$. Social media services are widely used, varied, and continually innovating, offering substantial opportunities for health communication [9]. Given these inherent advantages of social media, there has been rapid growth in the use of different social media services by health care providers and the general population to communicate information regarding cancer prevention, such as basic cancer knowledge, healthy lifestyles, and the importance of cancer screenings.

Previous research on the use of social media in cancer prevention has primarily focused on educational material [7]. In most previous studies, general information regarding cancer screening has been reported as a key component of cancer prevention education. However, a single study has addressed the detailed descriptions of bowel preparation for colorectal cancer screening $[7,10]$. Among these studies, cancer screening awareness and behavior for different cancers were not thoroughly examined [7]. Thus, more insight is needed to understand better the effects of social media on peoples' awareness and behavior concerning the screening for different cancers.

Despite the diversity of social media services, the previous studies mainly used specific types of social media, including Facebook, Twitter, and WeChat (a widely used social media app in China) [10-12]. Although one qualitative study reported that Facebook use helped promote breast cancer screening awareness [12], inconsistent effects of different social media services were found for the screening behavior for different cancer types [10,11]. Rosemary et al [11] showed that using Twitter did not improve specific behaviors regarding breast cancer prevention, including screening. In contrast, one study revealed an improvement in colorectal screening behavior through the use of WeChat [10]. However, the discussions are still too limited to provide comprehensive information to determine and compare the effects of social media on cancer screening awareness and behavior for different cancer types.

The variety of evolving social media services makes it necessary to examine how the general use of social media impacts cancer screening awareness and behavior for different cancer types. Moreover, most users of different social media services vary across racial and ethnic minority and socioeconomic groups. Only certain populations were included in previous prevention studies using specific types of social media [7,10-12]. Even less is known about the general population's social media use related to cancer screening awareness and behavior for different types of cancer.

This study aimed to assess how the general use of social media impacted people's cancer screening awareness and behavior for different cancer types in a nationally representative sample of US adults without a cancer diagnosis. In addition, because of the inherent heterogeneity of different cancer types between males and females, the gender-specific effects of social media use were examined. By evaluating social media use in the general population, this study furthers our understanding of the relationship between health communications using social media and health-promoting behaviors. 


\section{Methods}

\section{Data Sources}

This study adopted data from the National Cancer Institute (NCI) Health Information National Trends Survey (HINTS), including HINTS 5 Cycle $1(2017, \mathrm{n}=3285)$, HINTS 5 Cycle 2 (2018, $\mathrm{n}=3504)$, and HINTS 5 Cycle 3 (2019, n=5438). The HINTS is a nationwide survey of noninstitutionalized individuals aged 18 years and older in the United States and has been conducted every 1 to 2 years by the NCI since 2003. It uses a probability-based sampling with a 2-level stratified design, considering areas with high or low concentrations of minorities. Data from the 3 surveys were collected by mail or Web Pilot from January to May 2017, 2018, and 2019, respectively. Data were available in a publicly accessible repository that does not issue DOIs. Publicly available data sets were analyzed in this study. More detailed information on the study design and high-quality HINTS data have been published elsewhere [13] After excluding those individuals with a cancer diagnosis, 10,124 participants remained. Participants with missing data for any variables were excluded from the final analyses, resulting in a sample of 7090 social media users and 2775 social media nonusers.

\section{Measurements}

\section{Social Media Use}

Social media use was assessed using all 5 subquestions of the B14 question in the HINTS questionnaire ("Sometimes people use the internet to connect with other people online through social networks like Facebook or Twitter. This is often called 'social media."”). The 5 subquestions asked, "In the past 12 months, have you used the internet for any of the following reasons: (1) to visit a social networking site, such as Facebook or LinkedIn; (2) to share health information on social networking sites, such as Facebook or Twitter; (3) to write in an online diary or blog (ie, weblog); (4) to participate in an online forum or support group for people with similar health or medical issues; or (5) to watch a health-related video on YouTube?" The participants answered either "yes" or "no," and those using social media were defined by a "yes" response to any of the 5 questions.

\section{Cancer Screening Awareness and Behavior}

The measures of cancer screening awareness consisted of 3 dependent variables, which were assessed by 3 questions: (1) "Have you ever heard of the hepatitis $\mathrm{C}$ virus (also known as Hep C or HCV)?"; (2) "Have you ever heard of HPV?"; (3) "Before today, have you ever heard of the cervical cancer vaccine or HPV shot?" Participants indicated their response with either a "yes" (1) or "no" (0).

Cancer screening behavior measures contained 4 dependent variables defined by 4 questions: (1) "Have you ever had a prostate-specific antigen (PSA) test?" (1=yes; $0=$ no); (2) "How long ago did you have your most recent Papanicolaou (Pap) test to check for cervical cancer?" (1=I have had a Pap test [a year ago or less, 1 to 2 years ago, 2 to 3 years ago, 3 to 5 years ago, or more than 5 years ago]; $0=$ I have never had a Pap test); (3) "When did you have your most recent mammogram to check for breast cancer, if ever?" ( $1=$ I have had a mammogram [a year ago or less, 1 to 2 years ago, 2 to 3 years ago, 3 to 5 years ago, or more than 5 years ago]; $0=$ I have never had a mammogram); (4) "Have you ever had one of these tests, including colonoscopy, sigmoidoscopy, and stool blood test to check for colon cancer?" (1=yes; $0=$ no).

Due to the differences in the survey content between the different Cycles, the data for HCV were from Cycle 2 (2018) and Cycle 3 (2019), the data for HPV and the HPV vaccine were from Cycles 1 to 3 (2017-2019), the data for PSA testing were from Cycle 1 (2017) and Cycle 3 (2019), the data for Pap and breast cancer testing were from Cycles 1 to 3 (2017-2019), and the data for colon cancer testing were from Cycle 2 (2018) and Cycle 3 (2019).

\section{Sociodemographic Covariates}

The sociodemographic characteristics in this study included self-reported measures of gender (male or female), age, race and ethnicity (non-Hispanic White or racial and ethnic minority), education level (high school or less or more than high school), income (less than US $\$ 20,000$ or US $\$ 20,000$ or more annually), and geographic area (nonmetropolitan or metropolitan). Measurements of the geographic area were obtained by 9 metropolitan codes corresponding to the Rural-Urban Continuum Codes with a range of 1 to 9 [14]. Based on the cutoff points adopted by the United States Department of Agriculture and previous HINTS studies, this study divided the geographic areas into metropolitan (codes 1 to 3 ) and nonmetropolitan (codes 4 to 9) areas [14-16].

\section{Statistical Analysis}

This study compared the sociodemographic characteristics of social media users and nonusers via chi-squared tests for categorical variables and 2-tailed $t$-tests for continuous variables. Propensity-score matched analysis was carried out to adjust the sociodemographic variables between the social media users and nonusers. Confounders used for matching social media users and nonusers included gender, age, race and ethnicity, education, income, and geographic area. Given the gender differences for different cancer types, the differences in cancer screening awareness and behavior were compared by the social media use of males and females. Crude odds ratios (cOR), adjusted odds ratios (aOR), and their $95 \%$ CIs stratified by gender were computed using univariate and multivariable logistic regression analyses to clarify the impact of social media use on each dependent variable. This study adjusted the multivariable analyses for potential confounders, including gender, age, race and ethnicity, education, income, and geographic area.

All statistical analyses were carried out using SAS (version 9.4; SAS Institute). Given the complex survey design of the HINTS, jackknife replicate weights were incorporated into the analyses to obtain population-level estimates. The jackknife method creates a set of replicate samples from the original sample and provides an estimate of the variable and its variance of interest $[17,18]$. A $P<.05$ was considered statistically significant. 


\section{Results}

\section{Sociodemographic Characteristics and Social Media Use}

All percentages, means, and standard errors reported in this section are the weighted values. Among 10,124 participants without a diagnosis of cancer, $50 \%(n=4103)$ were male, and the average age was 48 (SD 0.20) years (Table 1). Nearly two-thirds ( $\mathrm{n}=5551,59 \%)$ of the participants were non-Hispanic White, and about one-third $(n=2496,31 \%)$ had a high school education or less. The income of about $18 \%(n=1715)$ of the participants was less than US \$20,000 annually. In addition, $14 \%(n=1246)$ of the population lived in nonmetropolitan areas. Among the 9868 participants with complete information, 72\% $(n=7093)$ used social media. There were statistically significant differences in all sociodemographic characteristics between the social media user versus nonuser participants (all $P<.001$ ). Compared to social media nonusers, the social media users were more likely to be female $(56 \%, \mathrm{n}=1291)$, tended to be younger (mean age 44 years vs 59 years), non-Hispanic White $(n=4129$, $61 \%$ vs $n=1344,54 \%$ ), were educated above a high school level ( $76 \%, n=5765$ vs $\mathrm{n}=1580,50 \%)$, and were more likely to live in metropolitan areas $(n=6298,88 \%$ vs $n=2361,83 \%)$.

Table 1. Sociodemographic characteristics for social media users and nonusers. All percentages, means, and standard errors reported in the table are the weighted values.

\begin{tabular}{|c|c|c|c|c|}
\hline \multirow[t]{2}{*}{ Characteristics } & \multirow[t]{2}{*}{ Total $(\mathrm{N}=10,124)$} & \multicolumn{3}{|c|}{ Social media use $(\mathrm{n}=9868)$} \\
\hline & & Use $(n=7093)$ & Nonuse $(n=2775)$ & $P$ value $^{\mathrm{a}}$ \\
\hline \multicolumn{5}{|l|}{ Gender, n (\%) } \\
\hline Male & $4103(49.4)$ & $2723(47.3)$ & $1291(56.4)$ & $<.001$ \\
\hline Female & $5895(50.6)$ & $4308(52.7)$ & 1433 (43.6) & \\
\hline Age (years), mean (SE) & $47.6(0.20)$ & $44.1(0.25)$ & $58.8(0.56)$ & $<.001$ \\
\hline \multicolumn{5}{|l|}{ Race and ethnicity, n (\%) } \\
\hline Non-Hispanic White & $5551(58.6)$ & $4129(60.7)$ & $1344(53.5)$ & $<.001$ \\
\hline Racial and ethnic minority & $4573(41.4)$ & $2964(39.3)$ & $1431(46.5)$ & \\
\hline \multicolumn{5}{|l|}{ Education, n (\%) } \\
\hline$\leq$ High school & $2496(30.7)$ & $1277(24.4)$ & $1108(49.6)$ & $<.001$ \\
\hline$>$ High school & 7479 (69.3) & 5765 (75.6) & $1580(50.4)$ & \\
\hline \multicolumn{5}{|l|}{ Income (US), n (\%) } \\
\hline$<\$ 20,000$ & $1715(18.0)$ & $909(14.9)$ & $732(27.6)$ & $<.001$ \\
\hline$\geq \$ 20,000$ & $7397(82.0)$ & $5662(85.1)$ & $1616(72.4)$ & \\
\hline \multicolumn{5}{|l|}{ Geographic area, n (\%) } \\
\hline Nonmetropolitan & $1246(13.5)$ & $795(12.4)$ & $414(17.5)$ & $<.001$ \\
\hline Metropolitan & $8878(86.5)$ & $6298(87.6)$ & $2361(82.5)$ & \\
\hline
\end{tabular}

${ }^{\mathrm{a}} \mathrm{Chi}$-squared tests for categorical variables and $t$-tests for continuous variables.

\section{Sociodemographics of Participants After Propensity-Score Matching}

After propensity-score matching, a total of 3794 (33\%) participants (1897 social media users and nonusers each) remained (Table 2). Of those 1864 (58\%) were male, 2110
(59\%) were non-Hispanic white, 1278 (45\%) had a high school education or less, 907 (24\%) had an annual income below US $\$ 20,000$, and $528(16 \%)$ lived in nonmetropolitan areas. No statistically significant differences between social media users and nonusers were found in any of the sociodemographic characteristics. 
Table 2. Sociodemographic characteristics between social media users and nonusers matched by the propensity-score method. All percentages, means, and standard errors reported in the table are the weighted values.

\begin{tabular}{|c|c|c|c|c|}
\hline \multirow[t]{2}{*}{ Characteristics } & \multirow[t]{2}{*}{ Total $(n=3794)$} & \multicolumn{3}{|c|}{ Social media use $(n=3794)$} \\
\hline & & Use $(n=1897)$ & Nonuse ( $\mathrm{n}=1897)$ & $P$ value $^{\mathrm{a}}$ \\
\hline \multicolumn{5}{|l|}{ Gender, n (\%) } \\
\hline Male & $1861(57.6)$ & $941(57.2)$ & $920(58.0)$ & .77 \\
\hline Female & $1933(42.4)$ & $956(42.8)$ & 977 (42.0) & \\
\hline Age (years), mean (SE) & $55.5(0.42)$ & $55.6(0.49)$ & $55.5(0.69)$ & .98 \\
\hline \multicolumn{5}{|l|}{ Race/ethnicity, n (\%) } \\
\hline Non-Hispanic White & $2110(59.1)$ & $1066(59.2)$ & $1044(59.0)$ & .93 \\
\hline Racial and ethnic minority & $1684(40.9)$ & $831(40.8)$ & $853(41.0)$ & \\
\hline \multicolumn{5}{|l|}{ Education, n (\%) } \\
\hline$\leq$ High school & $1278(45.3)$ & $656(46.8)$ & $622(43.9)$ & .33 \\
\hline$>$ High school & $2516(54.7)$ & $1241(53.2)$ & $1275(56.1)$ & \\
\hline \multicolumn{5}{|l|}{ Income (US), n (\%) } \\
\hline$<\$ 20,000$ & $907(23.5)$ & $442(23.9)$ & $465(23.0)$ & .74 \\
\hline$\geq \$ 20,000$ & $2887(76.5)$ & $1455(76.1)$ & $1432(77.0)$ & \\
\hline \multicolumn{5}{|l|}{ Geographic area, n (\%) } \\
\hline Nonmetropolitan & $528(16.4)$ & $287(17.2)$ & $241(15.6)$ & .42 \\
\hline Metropolitan & $3266(83.6)$ & $1610(82.8)$ & $1656(84.4)$ & \\
\hline
\end{tabular}

${ }^{\mathrm{a}} \mathrm{Chi}$-squared tests for categorical variables and t-tests for continuous variables.

\section{Cancer Screening Awareness and Behavior by Social Media Use and Gender}

Male social media users, compared to nonusers, were more likely to have heard of $\mathrm{HCV}(\mathrm{n}=394,92 \%$ vs $\mathrm{n}=321,84 \%$; $P=.02)$ and HPV (n=502, 56\% vs $\mathrm{n}=398,43 \% ; P=.003)$. However, awareness of the HPV vaccine was not significantly different between male social media users and nonusers $(\mathrm{n}=420$, $48 \%$ vs $\mathrm{n}=369,41 \%$, respectively; $P=.12$ ). For all 1861 male participants, including both social media users and nonusers, about $815(49 \%)$ had taken PSA tests $(P=.97)$, and $488(55 \%)$ of the social media users had taken colon cancer tests compared to $433(47 \%)$ social media nonusers ( $P=.09$; Table 3$)$.

For the 1933 female participants, the social media users, compared to nonusers, were more likely to have heard of HCV $(89 \%, \mathrm{n}=402$ vs $75 \%, \mathrm{n}=336 ; P<.001)$, and HPV $(72 \%, \mathrm{n}=683$ vs $56 \%, \mathrm{n}=566 ; P \leq .001)$, and HPV vaccine $(72 \%, \mathrm{n}=664$ vs $58 \%, \mathrm{n}=577 ; P \leq .001)$. In addition, $97 \%(\mathrm{n}=913)$ social media users and $94 \%(\mathrm{n}=921)$ nonusers had taken Pap tests $(P=.31)$, $87 \%(\mathrm{n}=849)$ social media users and $85 \%(\mathrm{n}=874)$ nonusers had taken breast cancer tests $(P=.43)$, and $66 \%(n=514)$ social media users and $64 \%(\mathrm{n}=479)$ nonusers had taken colon cancer tests $(P=.61)$. 
Table 3. Differences in cancer screening awareness and behavior by social media use and gender. All percentages, means, and standard errors reported in the table are the weighted values.

\begin{tabular}{|c|c|c|c|c|c|c|}
\hline \multirow[t]{3}{*}{ Characteristics } & \multicolumn{6}{|c|}{ Social media use } \\
\hline & \multicolumn{3}{|c|}{ Male $(\mathrm{n}=1861)$} & \multicolumn{3}{|c|}{ Female $(\mathrm{n}=1933)$} \\
\hline & Use $(n=941)$ & Nonuse $(\mathrm{n}=920)$ & $P$ value $^{\mathrm{a}}$ & Use (n=956) & Nonuse $(\mathrm{n}=977)$ & $P$ value \\
\hline \multicolumn{7}{|l|}{ Awareness } \\
\hline Heard of $\mathrm{HCV}^{\mathrm{b}}$ (yes), n (\%) & $394(91.7)$ & $321(83.5)$ & .02 & $402(89.3)$ & $336(75.0)$ & .002 \\
\hline Heard of $\mathrm{HPV}^{\mathrm{c}}$ (yes), n (\%) & $502(56.4)$ & $398(43.4)$ & .003 & $683(72.3)$ & $566(55.5)$ & $<.001$ \\
\hline Heard of HPV vaccine (yes), n (\%) & $420(48.0)$ & $369(40.5)$ & .12 & $664(71.7)$ & $577(57.7)$ & $<.001$ \\
\hline \multicolumn{7}{|l|}{ Behavior } \\
\hline Had PSA ${ }^{\mathrm{d}}$ test (yes), n (\%) & $427(49.0)$ & $388(49.2)$ & .97 & N/A & N/A & \\
\hline Had Pap e test (yes), n (\%) & N/A & N/A & & $913(96.5)$ & $921(94.1)$ & .31 \\
\hline Had breast cancer test (yes), n (\%) & N/A & N/A & & $849(87.1)$ & $874(85.0)$ & .43 \\
\hline Had colon cancer test (yes), n (\%) & $488(54.8)$ & $443(46.7)$ & .09 & $514(65.7)$ & $479(64.0)$ & .61 \\
\hline
\end{tabular}

${ }^{\mathrm{a}} \mathrm{Chi}$-squared tests for categorical variables and t-tests for continuous variables.

${ }^{\mathrm{b}} \mathrm{HCV}$ : hepatitis $\mathrm{C}$ virus.

${ }^{\mathrm{c}} \mathrm{HPV}$ : human papillomavirus.

${ }^{\mathrm{d}} \mathrm{PSA}$ : prostate-specific antigen.

${ }^{\mathrm{e}}$ Pap: Papanicolaou.

\section{Impact of Social Media Use on Awareness and Behavior of Cancer Screening}

Table 4 reports the relationship between social media use and cancer screening awareness and behavior in male and female participants presented by weighted analyses. Male social media users were more likely to have heard of $\mathrm{HCV}$ (cOR 2.17, 95\% CI 1.25-3.77; aOR 2.27, 95\% CI 1.29-3.98) and HPV (cOR $1.68,95 \%$ CI 1.20-2.36; aOR 1.82, 95\% CI 1.29-2.58) compared to nonusers. No statistically significant effects of social media use on awareness of the HPV vaccine and taking PSA tests or colon cancer tests were observed in males.

Table 4. Univariate and multivariable logistic regression assessing the impact of social media use on cancer screening awareness and behavior. Weighted analyses are presented.

\begin{tabular}{|c|c|c|c|c|c|c|c|c|}
\hline \multirow[t]{2}{*}{ Characteristics } & \multicolumn{4}{|l|}{ Male $(n=1861)$} & \multicolumn{4}{|l|}{ Female $(n=1933)$} \\
\hline & $\mathrm{cOR}^{\mathrm{a}}(95 \% \mathrm{CI})$ & $P$ value & $\mathrm{aOR}^{\mathrm{b}}(95 \% \mathrm{CI})$ & $P$ value & $\operatorname{cOR}(95 \% \mathrm{CI})$ & $P$ value & aOR $(95 \% \mathrm{CI})$ & $P$ value \\
\hline \multicolumn{9}{|l|}{ Awareness } \\
\hline Heard of $\mathrm{HCV}^{\mathrm{c}}$ & $2.17(1.25-3.77)$ & .007 & $2.27(1.29-3.98)$ & .005 & $2.80(1.57-4.99)$ & $<.001$ & $2.86(1.51-5.40)$ & .002 \\
\hline Heard of HPV ${ }^{\mathrm{d}}$ & $1.68(1.20-2.36)$ & .003 & $1.82(1.29-2.58)$ & .001 & $2.10(1.53-2.87)$ & $<.001$ & $2.35(1.65-3.33)$ & $<.001$ \\
\hline Heard of HPV vaccine & $1.35(0.92-1.98)$ & .12 & $1.42(0.98-2.06)$ & .07 & $1.85(1.34-2.57)$ & $<.001$ & $2.06(1.41-3.00)$ & $<.001$ \\
\hline \multicolumn{9}{|l|}{ Behavior } \\
\hline Had PSA $^{\mathrm{e}}$ test & $0.99(0.68-1.46)$ & .97 & $1.17(0.73-1.88)$ & .52 & N/A & & N/A & \\
\hline Had Pap ${ }^{\mathrm{f}}$ Test & N/A & & N/A & & $1.72(0.57-5.19)$ & .33 & $1.54(0.58-4.10)$ & .38 \\
\hline Had breast cancer test & N/A & & N/A & & $1.20(0.76-1.88)$ & .43 & $1.06(0.70-1.60)$ & .78 \\
\hline Had colon cancer test & $1.38(0.95-2.02)$ & .09 & $1.58(0.98-2.54)$ & .06 & $1.08(0.80-1.45)$ & .62 & $1.24(0.88-1.74)$ & .21 \\
\hline
\end{tabular}

\footnotetext{
${ }^{a}$ cOR: crude odds ratio.

$\mathrm{b}_{\mathrm{aOR}}$ : adjusted odds ratio.

${ }^{\mathrm{c}} \mathrm{HCV}$ : hepatitis $\mathrm{C}$ virus.

${ }^{\mathrm{d}} \mathrm{HPV}$ : human papillomavirus.

${ }^{\text {e}}$ PSA: prostate-specific antigen.

${ }^{\text {f}}$ Pap: Papanicolaou.
} 
Female social media users were more than twice as likely to have heard of HCV (cOR 2.80, 95\% CI 1.57-4.99; aOR 2.86, 95\% CI 1.51-5.40), HPV (cOR 2.10, 95\% CI 1.53-2.87; aOR $2.35,95 \%$ CI $1.65-3.33$ ) or the HPV vaccine (cOR $1.85,95 \%$ CI 1.34-2.57; aOR 2.06, 95\% CI 1.41-3.00) compared to nonusers. There was no statistically significant impact of social media use on taking Pap tests, breast cancer tests, and colon cancer tests.

\section{Discussion}

\section{Principal Results}

Our study conducted a comprehensive evaluation of the effects of general social media use on cancer screening awareness and behavior among US adults without a cancer diagnosis. Although the impact of social media on screening awareness varied across different cancer types and genders, we showed social media could promote cancer screening awareness in the general population. However, it did not improve screening behavior after adjusting for race and ethnicity and socioeconomic status. In light of the growing use of social media by health care providers and the general population, the findings of this study can contribute to targeted messaging for cancer prevention and reduce disparities between the different groups.

After matching and controlling for sociodemographic characteristics, we found that the general social media use had a significant impact on the awareness of HPV in both male and female adults. In contrast, its effect on HPV vaccine awareness was only significant in female adults. Similar to an online cervical cancer prevention study, HPV awareness can be increased through social media [19]. Persistent infection with specific HPV subtypes accounts for almost all cervical cancers in women, practically all anal cancers (over 9 out of 10), most vaginal, oropharyngeal, vulvar, and penile cancers (between two-thirds and three-quarters), and some oral cavity and laryngeal cancers in both men and women [20]. In addition to cervical cancer, most HPV-associated cancers could be reduced by current vaccines [20]. Hence, HPV vaccination is recommended for both males and females at specific ages. The American Cancer Society suggests that all children aged 11 or 12 years should be vaccinated against HPV infections. HPV vaccination with Gardasil 9 (Merck) has been approved for men and women aged 26 to 45 years by the United States Food and Drug Administration since 2018 [1,21]. An Australian study suggested that Twitter-derived measures of information exposure were associated with HPV vaccine coverage for both adolescent males and females [22]. Hence, in addition to communicating about HPV vaccination and cervical cancer prevention, social media can provide additional health information about other HPV-associated cancers and target the male population.

A significant impact of social media use on HCV awareness was also found for both male and female adults in this study. A southeast Michigan study on health disparities in hepatitis C revealed a suboptimal impact of multiple sociodemographic factors on hepatitis C screening and care [23]. Through the internet and a personal health record training program, health-related internet use increased among low-income patients with $\mathrm{HCV}$, and their self-efficacy and patient activation were also improved [24]. As a patient-driven internet service, social media emerges as a tool linking the general population with $\mathrm{HCV}$ awareness. However, its impact on improving hepatitis $\mathrm{C}$ screening and care among specific groups at high risk of $\mathrm{HCV}$ requires further research.

Social media use did not significantly impact cancer screening behavior, including taking PSA tests in male adults, Pap tests and breast cancer tests in female adults, or colon cancer tests in both male and female adults. In contrast, previous studies regarding specific social media services in the United States or China showed inconsistent effects of social media on cancer screening behavior [10-12]. These results may be due partly to misinformation in cancer communications commonly shared via social media.

There are increasing concerns about misleading or inaccurate medical science information on social media [25-27]. For example, a recent study on the accuracy of genitourinary malignancy articles shared on social media showed inaccurate and misleading articles were shared at a significantly higher rate on average than accurate articles, and 1 in 10 articles about PSA testing contained misinformation [28].

In addition, a lack of consensus on the clinical use of cancer screening and treatment may cause unnecessary panic when shared via social media. The so-called gray area of PSA testing with a high rate of false positives has resulted in its questionable clinical usefulness in Italy [29]. Prostate cancer tends to develop slowly, and its overdiagnosis and overtreatment are controversial [30]. Overdiagnosis and overtreatment have the potential for unnecessary side effects, including physical and psychological harms. The decrements in quality of life from side effects may offset some of the gains in length of life obtained from screening [31,32].

Given that social media amplifies scientific information and misinformation [25], a Japanese study [33] has revealed the information war for and against cancer screening messages has begun online. Therefore, accurate information with more engagement from scientists or professional institutions on social media is urgently needed to prevent misinformation about cancer screening from expanding and to achieve the screening target rates recommended in Healthy People 2030. In addition, collaboration with celebrities diagnosed with cancer may trigger substantial social media interest compared to traditional efforts for raising cancer screening awareness and affect screen behaviors, allowing health care providers to leverage social media methods like celebrities.

In terms of routine cancer care, PSA or colon cancer tests for males and Pap tests and breast cancer tests for females are part of routine check-ups. However, it may not be easy to change the behavior of people with negative perceptions regarding cancer screenings. As several HPV vaccine studies have pointed out, prior exposure to negative information was correlated with the later expression of negative opinions [34], and among Twitter users in Canada, the United Kingdom, and Australia, those with negative or opposing views about vaccinations were more likely to be better connected than those with positive views [35], leading to more barriers to improving routine cancer screening. As for cancers and tests that are not part of routine 
care, such as skin cancer [31], social media could be used to increase prior exposure to positive views for targeted populations to raise screening rates.

The findings in this study also suggest that among social media users, increased health information awareness did not lead to increased cancer screening behavior. The gap between "knowing" and "doing" in cancer screening behavior may be associated with the social characteristics of social media. Vaterlaus et al [36] reported a significant impact of social media on diet and exercise, which are commonly shared on social media. Compared to diet and exercise, health communication about cancer screening targets relatively small groups and has less engagement. Breast Cancer Awareness Month and associated events on Twitter revealed that a strategic communication plan calls for ongoing social media conversations on breast cancer and screening mammograms to help increase breast cancer screening rates [11].

The reach of social media is substantial, and the demographics of health-related users vary across different social media platforms [37]. It is valuable to promote cancer screening through various social media platforms according to the demographics of their health-related users to increase cancer screening rates for certain socioeconomic groups and hard-to-reach populations. Combined with ads targeted toward specific audiences, social media platforms could provide potentially innovative and effective ways to communicate positive health information.

\section{Limitations}

This study had several limitations. First, this study was based on the general use of social media rather than health-related use, which may result in measurement errors. Given the wide use of social media, it is difficult to separate health communications from the general use of social media. For instance, COVID-19 dominated social media communications at the beginning of 2020. Furthermore, the degree of social network use (eg, frequency and duration) was not quantified, and thus, the time-dependent effects of social media use could not be measured. Second, this study defined the use of Pap tests and breast cancer tests via "yes" or "no" responses among all the participants, without considering the recommended screening frequency by age. Moreover, the patterns of social media use vary across different age groups. Further studies on the effects of social media use on the frequency of cancer screening awareness and behavior at different ages should be conducted.

\section{Conclusions}

This study identified the association between social media use and cancer screening awareness and behavior among people without a cancer diagnosis after matching and controlling for sociodemographic factors. Social media use was associated with HPV and HCV awareness among both male and female participants, and the awareness of the HPV vaccine among female participants. No significant correlation was observed between social media use and screening behavior, including PSA, Pap, breast cancer, or colon cancer test-taking. This study provided a comprehensive assessment of the association between general social media use and cancer screening awareness and behavior for different types of cancer in the general population. Our study suggests that health communication using social media can effectively impact the awareness of specific cancers. Still, a more innovative and targeted approach is needed, including accurately delivering messages to hard-to-reach populations and improving specific screening behaviors.

\section{Acknowledgments}

This work was supported by the University of International Business and Economics (UIBE) Huiyuan outstanding young scholars research fund (grant 17YQ15) and the Fundamental Research Funds for the Central Universities in UIBE (grant CXTD10-10).

\section{Conflicts of Interest}

None declared.

\section{References}

1. Smith R, Andrews K, Brooks D, Fedewa S, Manassaram - Baptiste D, Saslow D, et al. Cancer screening in the United States, 2019: A review of current American Cancer Society guidelines and current issues in cancer screening. CA A Cancer J Clin 2019 Mar 15;69(3):184-210. [doi: 10.3322/caac.21557]

2. Siegel R, Miller KD, Jemal A. Cancer statistics, 2019. CA A Cancer J Clin 2019 Jan 08;69(1):7-34. [doi: 10.3322/caac.21551]

3. Islami F, Miller KD, Siegel RL, Zheng Z, Zhao J, Han X, et al. National and State Estimates of Lost Earnings From Cancer Deaths in the United States. JAMA Oncol 2019 Sep 12;5(9):e191460. [doi: 10.1001/jamaoncol.2019.1460]

4. White A, Thompson TD, White MC, Sabatino SA, de Moor J, Doria-Rose PV, et al. Cancer Screening Test Use - United States, 2015. MMWR Morb Mortal Wkly Rep 2017 Mar 03;66(8):201-206 [FREE Full text] [doi: 10.15585/mmwr.mm6608a1] [Medline: 28253225]

5. Goel MS, Wee CC, McCarthy EP, Davis RB, Ngo-Metzger Q, Phillips RS. Racial and ethnic disparities in cancer screening the importance of foreign birth as a barrier to care. J Gen Intern Med 2003 Dec;18(12):1028-1035. [doi: 10.1111/j.1525-1497.2003.20807.x]

6. Wender RC, Brawley OW, Fedewa SA, Gansler T, Smith RA. A blueprint for cancer screening and early detection: Advancing screening's contribution to cancer control. CA A Cancer J Clin 2018 Nov 19;69(1):50-79. [doi: 10.3322/caac. 21550$]$ 
7. Han CJ, Lee YJ, Demiris G. Interventions Using Social Media for Cancer Prevention and Management. Cancer Nurs 2018;41(6):E19-E31. [doi: 10.1097/ncc.0000000000000534]

8. Attai DJ, Cowher MS, Al-Hamadani M, Schoger JM, Staley AC, Landercasper J. Twitter Social Media is an Effective Tool for Breast Cancer Patient Education and Support: Patient-Reported Outcomes by Survey. J Med Internet Res 2015 Jul 30;17(7):e188. [doi: 10.2196/jmir.4721]

9. Huo J, Desai R, Hong Y, Turner K, Mainous AG, Bian J. Use of Social Media in Health Communication: Findings From the Health Information National Trends Survey 2013, 2014, and 2017. Cancer Control 2019 Apr 18;26(1):107327481984144. [doi: 10.1177/1073274819841442]

10. Kang X, Zhao L, Leung F, Luo H, Wang L, Wu J, et al. Delivery of Instructions via Mobile Social Media App Increases Quality of Bowel Preparation. Clinical Gastroenterology and Hepatology 2016 Mar;14(3):429-435.e3. [doi: 10.1016/j.cgh.2015.09.038]

11. Thackeray R, Burton SH, Giraud-Carrier C, Rollins S, Draper CR. Using Twitter for breast cancer prevention: an analysis of breast cancer awareness month. BMC Cancer 2013 Oct 29;13(1). [doi: 10.1186/1471-2407-13-508]

12. Abramson K, Keefe B, Chou WS. Communicating About Cancer Through Facebook: A Qualitative Analysis of a Breast Cancer Awareness Page. Journal of Health Communication 2014 Dec 13;20(2):237-243. [doi: 10.1080/10810730.2014.927034]

13. Finney RL, Blake K, Skolnick V, Davis T, Moser R, Hesse B. Data resource profile: the national Cancer Institute's Health Information National Trends Survey (HINTS). International journal of epidemiology 2019:17-17j. [doi: 10.1093/ije/dyz083]

14. United SDOA. Rural-urban continuum codes: Documentation. Rural-Urban Continuum Codes. 2013. URL: https://www. ers.usda.gov/data-products/rural-urban-continuum-codes/documentation.aspx [accessed 2020-04-25]

15. Vereen RN, Westmaas JL, Bontemps-Jones J, Jackson K, Alcaraz KI. Trust of Information about Tobacco and E-Cigarettes from Health Professionals versus Tobacco or Electronic Cigarette Companies: Differences by Subgroups and Implications for Tobacco Messaging. Health Communication 2018 Nov 13;35(1):89-95. [doi: 10.1080/10410236.2018.1544875]

16. Heffner JL, Mull KE. Smartphone Ownership Among US Adult Cigarette Smokers: 2014 Health Information National Trends Survey (HINTS) Data. J Med Internet Res 2017 Aug 31;19(8):e305. [doi: 10.2196/jmir.7953]

17. Chandrasekaran R, Katthula V, Moustakas E. Patterns of Use and Key Predictors for the Use of Wearable Health Care Devices by US Adults: Insights from a National Survey. J Med Internet Res 2020 Oct 16;22(10):e22443. [doi: 10.2196/22443]

18. Lavrakas PJ. Jackknife variance estimation. In: Encyclopedia of Survey Research Methods. Newbury Park, California: Sage publications; 2008. [doi: 10.4135/9781412963947.n257]

19. Lyson HC, Le GM, Zhang J, Rivadeneira N, Lyles C, Radcliffe K, et al. Social Media as a Tool to Promote Health Awareness: Results from an Online Cervical Cancer Prevention Study. J Canc Educ 2018 Jun 11;34(4):819-822. [doi: 10.1007/s13187-018-1379-8]

20. Saraiya M, Unger E, Thompson T, Lynch C, Hernandez B, Lyu C. US assessment of HPV types in cancers: implications for current and 9-valent HPV vaccines. JNCI: Journal of the National Cancer Institute 2015;107(6):djv086. [doi: 10.1093/jnci/djv086]

21. Saslow D, Andrews KS, Manassaram-Baptiste D, Loomer L, Lam KE, Fisher-Borne M, et al. Human papillomavirus vaccination guideline update: American Cancer Society guideline endorsement. CA: A Cancer Journal for Clinicians 2016 Jul 19;66(5):375-385. [doi: 10.3322/caac.21355]

22. Dyda A, Shah Z, Surian D, Martin P, Coiera E, Dey A, et al. HPV vaccine coverage in Australia and associations with HPV vaccine information exposure among Australian Twitter users. Human Vaccines \& Immunotherapeutics 2019 Apr 12;15(7-8):1488-1495. [doi: 10.1080/21645515.2019.1596712]

23. Bourgi K, Brar I, Baker-Genaw K. Health Disparities in Hepatitis C Screening and Linkage to Care at an Integrated Health System in Southeast Michigan. PLoS ONE 2016 Aug 15;11(8):e0161241. [doi: 10.1371/journal.pone.0161241]

24. McInnes D, Solomon J, Shimada S, Petrakis B, Bokhour B, Asch S. Developmentevaluation of an internetpersonal health record training program for low-income patients with HIV or hepatitis C. Medical care 2013:S62-S66. [doi: 10.1097/mlr.0b013e31827808bf]

25. Merchant RM, Asch DA. Protecting the Value of Medical Science in the Age of Social Media and "Fake News". JAMA 2018 Dec 18;320(23):2415-2416. [doi: 10.1001/jama.2018.18416]

26. Chou WS, Oh A, Klein WMP. Addressing Health-Related Misinformation on Social Media. JAMA 2018 Dec 18;320(23):2417-2418. [doi: 10.1001/jama.2018.16865]

27. Wang Y, McKee M, Torbica A, Stuckler D. Systematic Literature Review on the Spread of Health-related Misinformation on Social Media. Social Science \& Medicine 2019 Nov;240:112552. [doi: 10.1016/j.socscimed.2019.112552]

28. Alsyouf M, Stokes P, Hur D, Amasyali A, Ruckle H, Hu B. 'Fake News' in urology: evaluating the accuracy of articles shared on social media in genitourinary malignancies. BJU Int 2019 May 13;124(4):701-706. [doi: 10.1111/bju.14787]

29. Perdonà S, Cavadas V, Di Lorenzo G, Damiano R, Chiappetta G, Del Prete P, et al. Prostate Cancer Detection in the "Grey Area" of Prostate-Specific Antigen Below $10 \mathrm{ng} / \mathrm{ml}$ : Head-to-Head Comparison of the Updated PCPT Calculator and Chun's Nomogram, Two Risk Estimators Incorporating Prostate Cancer Antigen 3. European Urology 2011 Jan;59(1):81-87. [doi: 10.1016/j.eururo.2010.09.036] 
30. Loeb S, Bjurlin MA, Nicholson J, Tammela TL, Penson DF, Carter HB, et al. Overdiagnosis and Overtreatment of Prostate Cancer. European Urology 2014 Jun;65(6):1046-1055. [doi: 10.1016/j.eururo.2013.12.062]

31. Falzone AE, Brindis CD, Chren M, Junn A, Pagoto S, Wehner M, et al. Teens, Tweets, and Tanning Beds: Rethinking the Use of Social Media for Skin Cancer Prevention. American Journal of Preventive Medicine 2017 Sep;53(3):S86-S94. [doi: 10.1016/j.amepre.2017.04.027]

32. Heijnsdijk EA, Wever EM, Auvinen A, Hugosson J, Ciatto S, Nelen V, et al. Quality-of-Life Effects of Prostate-Specific Antigen Screening. N Engl J Med 2012 Aug 16;367(7):595-605. [doi: 10.1056/nejmoa1201637]

33. Okuhara T, Ishikawa H, Okada M, Kato M, Kiuchi T. Assertions of Japanese Websites for and Against Cancer Screening: a Text Mining Analysis. Asian Pac J Cancer Prev 2017 Apr 01;18(4):1069-1075 [FREE Full text] [doi: 10.22034/APJCP.2017.18.4.1069] [Medline: 28547943]

34. Dunn AG, Leask J, Zhou X, Mandl KD, Coiera E. Associations Between Exposure to and Expression of Negative Opinions About Human Papillomavirus Vaccines on Social Media: An Observational Study. J Med Internet Res 2015 Jun 10;17(6):e144. [doi: 10.2196/jmir.4343]

35. Shapiro GK, Surian D, Dunn AG, Perry R, Kelaher M. Comparing human papillomavirus vaccine concerns on Twitter: a cross-sectional study of users in Australia, Canada and the UK. BMJ Open 2017 Oct 05;7(10):e016869 [FREE Full text] [doi: 10.1136/bmjopen-2017-016869] [Medline: 28982821]

36. Vaterlaus JM, Patten EV, Roche C, Young JA. \#Gettinghealthy: The perceived influence of social media on young adult health behaviors. Computers in Human Behavior 2015 Apr;45:151-157. [doi: 10.1016/j.chb.2014.12.013]

37. Sadah SA, Shahbazi M, Wiley MT, Hristidis V. A Study of the Demographics of Web-Based Health-Related Social Media Users. J Med Internet Res 2015 Aug 06;17(8):e194. [doi: 10.2196/jmir.4308]

\author{
Abbreviations \\ aOR: adjusted odds ratios \\ cOR: crude odds ratios \\ HCV: hepatitis C virus \\ HINTS: Health Information National Trends Survey \\ HPV: human papillomavirus \\ NCI: National Cancer Institute \\ Pap: Papanicolaou \\ PSA: prostate-specific antigen \\ UIBE: University of International Business and Economics
}

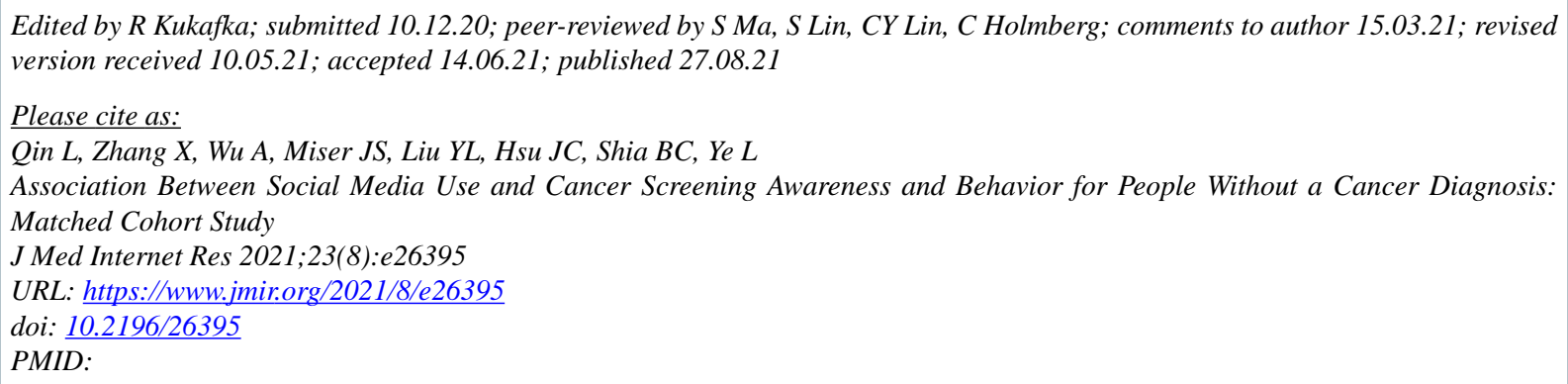

CLei Qin, Xiaomei Zhang, Anlin Wu, James S Miser, Yen-Lin Liu, Jason C Hsu, Ben-Chang Shia, Linglong Ye. Originally published in the Journal of Medical Internet Research (https://www.jmir.org), 27.08.2021. This is an open-access article distributed under the terms of the Creative Commons Attribution License (https://creativecommons.org/licenses/by/4.0/), which permits unrestricted use, distribution, and reproduction in any medium, provided the original work, first published in the Journal of Medical Internet Research, is properly cited. The complete bibliographic information, a link to the original publication on https://www.jmir.org/, as well as this copyright and license information must be included. 